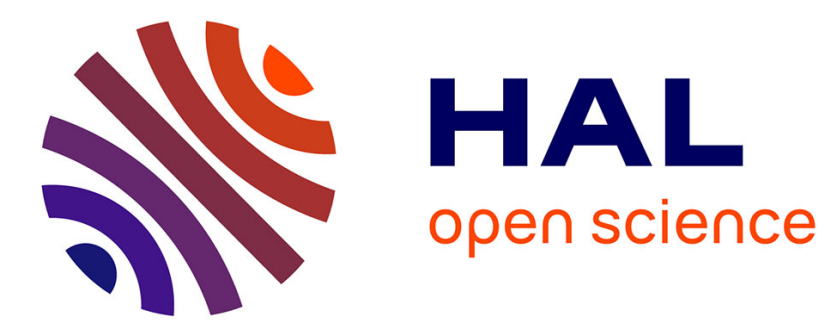

\title{
Multi-Flow Transmission and Carrier Aggregation Inter-Operation in HSPA+ Advanced
}

\author{
Ahlem Khlass, Salah Eddine Elayoubi, Thomas Bonald
}

\section{To cite this version:}

Ahlem Khlass, Salah Eddine Elayoubi, Thomas Bonald. Multi-Flow Transmission and Carrier Aggregation Inter-Operation in HSPA+ Advanced. VTC, Sep 2014, Vancouver, Canada. pp.1 - 5, 10.1109/VTCFall.2014.6966063 . hal-01112967

\section{HAL Id: hal-01112967 https://hal-imt.archives-ouvertes.fr/hal-01112967}

Submitted on 4 Feb 2015

HAL is a multi-disciplinary open access archive for the deposit and dissemination of scientific research documents, whether they are published or not. The documents may come from teaching and research institutions in France or abroad, or from public or private research centers.
L'archive ouverte pluridisciplinaire HAL, est destinée au dépôt et à la diffusion de documents scientifiques de niveau recherche, publiés ou non, émanant des établissements d'enseignement et de recherche français ou étrangers, des laboratoires publics ou privés. 


\section{Multi-flow Transmission and Carrier Aggregation Inter-operation in HSPA+ Advanced}

\author{
Ahlem Khlass \\ Orange Labs \& Telecom ParisTech \\ France \\ ahlem.khlass@orange.com
}

\author{
Salah Eddine Elayoubi \\ Orange Labs \\ Issy-Les-Moulineaux, France \\ salaheddine.elayoubi@orange.com
}

\author{
Thomas Bonald \\ Telecom ParisTech \\ Paris, France \\ thomas.bonald@telecom-paristech.fr
}

\begin{abstract}
Carrier aggregation and multi-flow transmission are among the most important features of HSPA+. While the former allows users to be served simultaneously by several carriers in the same sector, the latter enables adjacent sectors to simultaneously schedule different data streams to the same user in their overlapping region. In this paper, we investigate the inter-operation of these two features. We evaluate the flow-level performance using a method based on network simulation coupled with Markov chain analysis. Results in single-carrier mode show an improvement in throughput at low load and an efficient load balancing across sectors at high load. In multi-carrier mode, we show that coordination is no more recommended since it does not achieve any throughput gain over the classical multi-carrier system. This is due to the actual status of the standard that limits the number of carriers that can be used for the multi-flow transmission to two. However, if this restriction is released in the standard, our results show that multiflow transmission would bring significant gains.
\end{abstract}

Index Terms-HSPDA, Multi-flow Transmission, Carrier Aggregation, Flow Level Modeling, Queuing Theory.

\section{INTRODUCTION}

Facing the important increase of data traffic and the need for higher data rates, the third generation $(3 \mathrm{G})$ wireless technologies are still upgraded with new features that aim to improve both the users' experience and the operators' business.

In one side, Carrier aggregation (CA) has been introduced in High Speed Packet Access (HSPA) release 8 where two carrier components (CC) of $5 \mathrm{MHz}$ are used to expand the effective system bandwidth. The advantages of this concept is to improve the system capacity and to increase the user throughput anywhere in the cell, even at cell edge, regardless of the load conditions as shown in several studies as [1] [2] [3]. Indeed, with Dual-carrier HSPA, peak data rates up to $42 \mathrm{Mbps}$ in the downlink and $28 \mathrm{Mbps}$ in the uplink can be achieved, in addition to a pooling gain of 20-30\% [4] [5]. Therefore, this feature has received high attention from operators that want to improve their spectral efficiency, while delaying the deployment of new LTE system.

The concept of Dual-carrier HSDPA (DC-HSDPA) has been extended to three-carrier HSDPA in release 10 and generalized in release 11 [6] into Multi-carrier HSDPA (MC-HSDPA) with the utilization of up to four to carrier simultaneously.

Release 11 brought to HSDPA another major feature that is Multi-flow transmission [6]. This feature enables a user equipment (UE) in overlapping region between two sectors (belonging or not to the same NodeB) to be scheduled simultaneously by both. The main motivations are the improvement of cell edge user experience which suffers from very low rates due to the inter-cell interference, balancing the load across differently loaded cells and increasing robustness since the same data block is received from different cells [7] [8].

The main difference between multi-carrier and Multi-point HSDPA that should be highlighted is that the former allows multiple carriers, belonging to the same sector, to serve simultaneously the same UE, while the latter coordinates different sectors to transmit simultaneously to the same UE on the same carrier or different carriers.

Note that, in this paper, we will use the terms cells and sectors interchangeably, while the term carrier will refer to a frequency carrier of $5 \mathrm{MHz}$.

Although multi-flow transmission can be performed between sectors of the same NodeB or between different NodeBs, the former option is expected to be deployed first as it does not induce delay and bandwidth constraints on the backhaul. We thus focus in this paper on intra-NodeB multiflow and show how it performs when jointly deployed with carrier aggregation. Indeed, while previous works focused on the performance of multi-flow transmission in a single carrier setting [9] [10] [11], the interplay of multi-flow and carrier aggregation has not been studied yet. Indeed, the actual HSPA standard (Release 11) does not allow to perform multi-flow on more than 2 carriers and Release 12 does not bring any enhancement on this feature. We thus address in this work the following questions:

1) What is the expected performance from multi-flow HSDPA in terms of user throughput and load balancing ?

2) Do we need multi-flow transmission in multi-carrier HSDPA ?

We adopt for the performance evaluation a hybrid analytical/simulation approach in order to be able to assess the flow level performance. In fact, most of the existing works on cell coordination focus on the system level simulations based on a full-buffer traffic model, as proposed by 3GPP [12], with a high accuracy with respect to the lower layers (Physical and MAC layers), but do not consider the dynamic behavior of traffic. We thus use full buffer network simulations in order to model the achieved throughputs in the different positions of 
the network, coupled with a Markovian analysis that captures the flow-level dynamics of users.

This paper is organized as follows. In section II, we introduce the multi-flow transmission principles. In section III, we present the model. Performance evaluation under a variety of network settings and load situations are provided in section IV. Finally, conclusions and further work are drawn in section VI.

\section{MULTI-FLOW TRANSMISSION PRINCIPLES}

Multi-flow (MF) HSDPA has been proposed in 3GPP standard to improve users experience and to balance load between neighboring cells. It enables adjacent sectors to coordinate their transmission by sending different data blocks simultaneously to the same UE in the overlapping area.

We focus in this paper on intra-NodeB coordination as it is simpler to implement on top of legacy HSDPA networks and consider 4 multi-flow scenarios with respect to multi-flow and carrier aggregation implementation:

- Scenario 1: Baseline MF, an HSDPA network with a single deployed carrier implementing multi-flow (also named Single Frequency-Dual Cell (SF-DC) aggregation). This scenario is used to illustrate the basic performance of multi-flow transmission.

- Scenario 2: Baseline MF+CA, 4 HSDPA carriers are deployed and both carrier aggregation and multi-flow transmission are activated. In this case, users in the coordination area will use multi-flow transmission on two carriers (each sector will schedule two independent transport blocks), while other users will implement legacy carrier aggregation on 4 carriers. This scenario is the best multi-flow case allowed in the standard [6].

- Scenario 3: CA only, 4 HSDPA carriers are deployed and the operator chooses to activate carrier aggregation and deactivate multi-flow. In this case, each UE receives simultaneously 4 independent transport blocks from its serving sector.

- Scenario 4: MF+CA on 4 carriers, a network with 4 deployed carriers where carrier aggregation and multiflow are enabled on all carriers. In this case, a user in the coordination area will receive 8 independent transport blocks from the two sectors simultaneously. Note that this scenario is not allowed by the actual standard.

\section{MODEL}

\section{A. Radio aspects}

1) SINR analysis: We investigate the Signal-to-Noise-plusInterference-Ratio (SINR) and the system capacity in the following two cases.

a) Classical Single-Frequency HSDPA: Each UE $u$ is only associated to its serving BS $s$, the SINR is given by:

$$
\mathrm{SINR}_{u, s}=\frac{P_{u, s}}{\sum_{k \neq s} P_{u, k}+I_{u}+N},
$$

where $P_{u, k}$ denotes the power received by UE $u$ from BS $k$ of the same site, $I_{u}$ is the inter-site interference received by

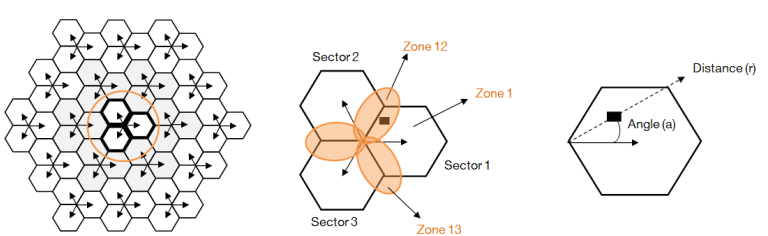

Fig. 1. Network model, site model, sector model.

UE $u$ and $N$ is the thermal noise. Considering a Gaussian channel, the Shannon capacity is given by:

$$
C^{S F}=W \log \left(1+\operatorname{SINR}_{u, s}\right)
$$

where $W$ is the channel bandwidth.

b) Multi-flow transmission: When multi-flow operation is activated, UE $u$ receives simultaneously two different transport blocks from the serving BS $s$ and from an adjacent BS $c$ on the same frequency, leading to two flows with respective SINRs:

$$
\mathrm{SINR}_{u, s}=\frac{P_{u, s}}{\epsilon P_{u, c}+\sum_{k \neq s, c} P_{u, k}+I_{u}+N}
$$

and

$$
\operatorname{SINR}_{u, c}=\frac{P_{u, c}}{\epsilon P_{u, s}+\sum_{k \neq s, c} P_{u, k}+I_{u}+N} .
$$

where $\epsilon$ denotes the receiver capability of canceling the interference from the other sectors within a given site. The Shannon capacity of a Gaussian channel is then given by

$$
C^{S F-D C}=W \log \left(1+\mathrm{SINR}_{u, s}\right)+W \log \left(1+\mathrm{SINR}_{u, c}\right)
$$

which is higher than that of the classical SF-HSDPA.

When carriers are aggregated, the system capacity can be derived from previous expressions by multiplying by the number of carriers.

2) Transmission rates: We consider a network of 20 homogeneous tri-sector sites as shown in figure 1. We focus on a given sector in the central site. Each position is referred by a distance from the site center $r$ and the angle relative to the main direction of the antenna $a$.

We use link level curves that associate to each SINR measured at a given position of a reference sector the corresponding throughput as described in [13]. We consider a fixed coordination threshold $\delta$, so that the coordination region represents $30 \%$ of the total sector surface. Moreover, we assume that the 3i-type receiver [6] is able to cancel perfectly the interference from the coordinated BSs, so that $\epsilon=0$. This gives an upper bound for MF performance.

We obtain four matrices of the transmission rates $R$ in different position $(a, r)$ relative to each scenario detailed in section II. The results are illustrated in figure 2 over a half-sector (PA3 channel, dense urban area with an inter-site distance of 500 meters).

For single-carrier mode, it is obvious that in order to boost users rates in cell edge, it is better to enable multi-flow operation for users in the coordination region. 


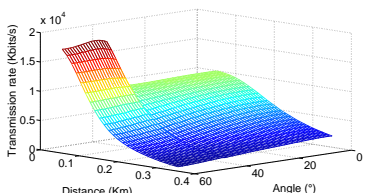

(a) Scenario 1

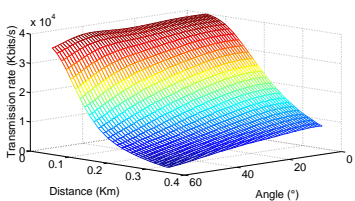

(c) Scenario 3

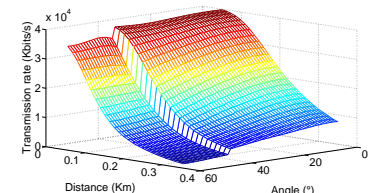

(b) Scenario 2

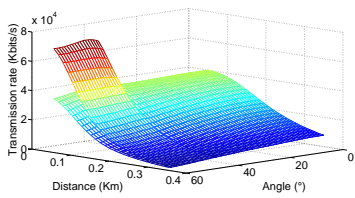

(d) Scenario 4
Fig. 2. Transmission rates.

However, in multi-carrier mode, it is more efficient to deploy carrier aggregation (with four carriers) for all users in the cell rather than multi-flow transmission for users in the coordination region where only two carriers can be aggregated.

Nevertheless, if this restriction is released, the performance will be more interesting since we get full benefit from both features. These results provide insights on the achievable rates, the performance in dynamic network settings considering users arrival and departure is given in the following.

\section{B. Resource Allocation Algorithm}

We consider a centralized scheduler for all the available frequencies based on a simple algorithm like Round-Robin where users are selected in each sector $S_{k}$ for $k=1 . .3$ at random independently of their channel variations. This is equivalent to a proportional fair scheduler when the channel variations are too slow or too fast to be exploited.

Users can be clustered into two zones:

- Non-coordination zones where users are served exclusively by a single $B S$ noted $Z_{1}, Z_{2}$ and $Z_{3}$.

- Coordination zones where users are served simultaneously by two $B S$ s noted $Z_{12}, Z_{23}$ and $Z_{31}$.

For instance, zone $Z_{1} \subset S_{1}$ includes users served exclusively by $B S_{1}$ and zone $Z_{12} \subset S_{1} \cap S_{2}$ includes users served simultaneously by $B S_{1}$ and $B S_{2}$ as illustrated in figure 1 . Let $\mathcal{K}=\{1,12,2,23,3,31\}$ be the set of zones in the site.

1) Single-carrier mode: Resources are divided between users so that $B S_{1}, B S_{2}$ and $B S_{3}$ can serve simultaneously users in zone $Z_{1}, Z_{2}$ and $Z_{3}$, respectively on the same frequency $f_{0}$. But, when to serve users in zone $Z_{12}$ for instance, $B S_{1}$ and $B S_{2}$ join their resources and block users from $Z_{1}$ and $Z_{2}$, similarly for users in zones $Z_{23}$ and $Z_{31}$.

We denote by $z_{k}(t)$ the number of users in each zone and $\vec{z}(t)$ their corresponding vector. The total number of users in the site is given by $\bar{z}$. Whenever $\vec{z}(t)=\vec{z}$, the scheduler selects UEs in zone $Z_{12}$ for instance a fraction of time

$$
\phi_{12}^{S C}(\vec{z})=\frac{z_{12}}{\bar{z}}+\frac{z_{3}}{\bar{z}} \frac{z_{12}}{z_{1}+z_{2}+z_{12}} .
$$

In fact, a user in zone $Z_{12}$ is served if it is selected at first with a probability of $z_{12} / \bar{z}$ or after selecting a user in zone
$Z_{3}$ with a probability of $z_{3} / \bar{z}$ (blocking by this way users in $Z_{23}$ and $Z_{13}$ ). Similarly, we get the fraction of time allocated to users in $Z_{1}$ :

$$
\phi_{1}^{S C}(\vec{z})=\frac{z_{1}+z_{23}}{\bar{z}}+\frac{z_{2}}{\bar{z}} \frac{z_{1}+z_{3}}{z_{1}+z_{3}+z_{31}}+\frac{z_{3}}{\bar{z}} \frac{z_{1}+z_{2}}{z_{1}+z_{2}+z_{12}},
$$

and so on for the other zones.

2) Multi-carrier mode: Let $F=\left\{f_{i}\right\}_{i=1, \ldots, 4}$ denotes the set of available frequencies in each sector and $F_{c} \subset F$ the set of frequencies that can be used for coordination. Recall that the actual status of the standard limits the number of carriers that can be used for multi-flow transmission to 2 .

Contrarily to the single-carrier mode, serving a user in the coordination zone ( $Z_{12}$ for instance) on the carriers in $F_{c}$ does not block all resources for the users in the non-coordination zone which can be served on the remaining carriers $F \backslash F_{c}$. Hence, users in $Z_{1}$ are served a fraction of time of:

$$
\phi_{1}^{F}(\vec{z})=\phi_{1}^{S C}(\vec{z})
$$

and

$\phi_{1}^{F \backslash F_{c}}(\vec{z})=\frac{z_{12}+z_{31}}{\bar{z}}+\frac{z_{2}}{\bar{z}} \frac{z_{31}}{z_{1}+z_{3}+z_{31}}+\frac{z_{3}}{\bar{z}} \frac{z_{12}}{z_{1}+z_{2}+z_{12}}$ on the carriers in $F$ and $F \backslash F_{c}$, respectively. For users in zone $Z_{12}$, they are selected a fraction of time of:

$$
\phi_{12}^{F_{c}}(\vec{z})=\phi_{12}^{S C}(\vec{z})
$$

using the set of carriers $F_{c}$; and so on for the other zones.

\section{Markov Analysis}

Let $\mathcal{C}=\{1,2, \ldots, N\}$ be the set of elementary surfaces such as each element $i \in \mathcal{C}$ corresponds to a particular position in the site and belongs to one of the six zones defined previously. We assume the traffic demand is uniformly distributed in the site. Users arrive in the network according to a Poisson process of intensity $\lambda$ and leave the network once they finish their data transfer. Precisely, users in class- $i$ of sector $S_{k}$ arrive following a Poisson process of intensity $\lambda_{i}$.

Flows are characterized by their size which is independent and identically distributed with mean $\sigma$; they contribute to the traffic with intensity $\lambda_{i} \sigma$. The class $i$ mean service rate is given by:

$$
\mu_{i}=R_{i} / \sigma
$$

where $R_{i}$ is the transmission rate represented in figure III-A2.

The system can be described as a Markov process [14]. Each state is denoted by $\vec{x}(t)=\left\{x_{i}(t)\right\}_{i \in \mathcal{C}}$ where $x_{i}(t)$ denotes the number of active flows in class $i$. Let $Q$ denotes the transition rate matrix. In the single-carrier mode, the transition rate between two states $\vec{x}$ and $\vec{x}^{\prime}$ is given by

$$
q^{S C}\left(\vec{x}, \vec{x}^{\prime}\right)= \begin{cases}\lambda_{i} & \text { for } \vec{x}^{\prime}=\vec{x}+\vec{e}_{i} \\ \frac{x_{i}}{z_{k}} \mu_{i} \phi_{k}^{S C}(z) & \text { for } \vec{x}^{\prime}=\vec{x}-\vec{e}_{i}, \\ i \in Z_{k}, k \in \mathcal{K}\end{cases}
$$

where $\vec{e}_{i}$ denotes an $N$-dimensional vector where the $i$ 'th component is equal to one and all the others are equal to zero. 
For the multi-carrier mode with 4 carriers, the transition rates are:

$$
\begin{aligned}
& q^{4 C}\left(\vec{x}, \vec{x}^{\prime}\right)= \\
& \begin{cases}\lambda_{i} & \text { for } \vec{x}^{\prime}=\vec{x}+e_{i} \\
\frac{x_{i}}{z_{k}} \mu_{i}\left(\phi_{k}^{F}(z)+\frac{1}{2} \phi_{k}^{F \backslash F_{c}}(z)\right) & \text { for } \vec{x}^{\prime}=\vec{x}-e_{i} \\
& \text { with } i \in Z_{1}, Z_{2}, Z_{3} \\
\frac{x_{i}}{z_{k}} \mu_{i} \phi_{k}^{F_{c}}(z) & \text { for } \vec{x}^{\prime}=\vec{x}-e_{i} \\
& \text { with } i \in Z_{12}, Z_{23}, Z_{31} .\end{cases}
\end{aligned}
$$

In fact, when four carriers are available, the mean service rate $\mu_{i}$ can only be achievable with the aggregation of 4 carriers; therefore, when users are served with the set $F \backslash F_{c}$ (containing only 2 carriers), the corresponding mean service rate is $\mu_{i} / 2$. Note that the sets of frequencies do not change during all the simulation time.

We can obtain the steady-state probabilities $\pi(\vec{x})$ by solving directly $\pi Q=0$ or by simulation. Then, the average numbers of users in each class $i$ is :

$$
E\left(x_{i}\right)=\sum x_{i} \pi(\vec{x})
$$

Using Little's law, we obtain that the mean delay of users in class $i$ :

$$
E\left(T_{i}\right)=\frac{E\left(x_{i}\right)}{\lambda_{i}}
$$

The throughput, defined as the ratio of the mean flow size to the mean flow delay, is given by

$$
\gamma_{i}=\sigma \frac{\lambda_{i}}{E\left(x_{i}\right)}
$$

which is used in the following, to evaluate the performance of multi-flow operation in the both single-carrier and multicarrier mode.

\section{Performance eVAluation}

Recall that we only consider intra-site coordination where the multi-flow is only performed between sectors within the same site. Performance is evaluated in terms of flowthroughput by injecting the peak rates obtained in section III-A2 in the Markovian flow-level analysis described in section IV for each scenario.

Note that, in addition to the data rate improvement, multiflow is expected to balance load across sectors; hence results are given for different load conditions.

\section{A. Scenario 1 performance analysis}

Figure 3 represents the flow throughput observed in the coordination and non-coordination zones according to scenario 1 (baseline MF) for different traffic intensities in comparison to the classical SC-HSDPA. We see that the multi-flow transmission is enable to increase the average throughput of around $20 \%$ at low load of at the expense of a decrease in the stability region of almost the same percentage. This can be explained by the fact that at high load, each sector has to handle not only the traffic from its own users but also that from sector coordination. Therefore, the multi-flow is only beneficial at low load.

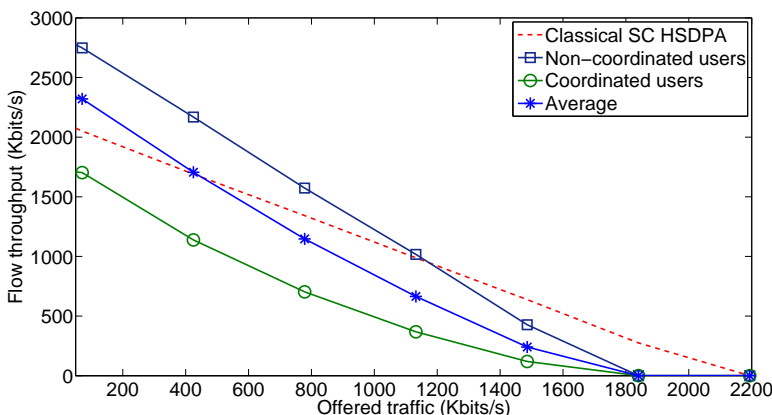

Fig. 3. Flow throughput vs. traffic (scenario 1).

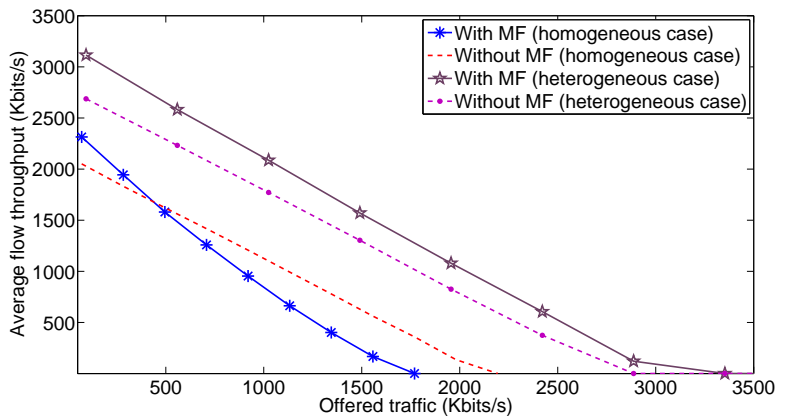

Fig. 4. Average throughput under different load conditions (scenario 1).

We consider now the case of heterogeneous load across sectors. We focus on a given sector and we suppose that the neighboring ones are relatively less loaded. The performance results are given in figure 4 in terms of average throughput. We note that in addition to the considerable throughput improvement, the system stability is improved when neighboring sectors are less loaded. Indeed, the multi-flow operation enables to offload the traffic from the highly to lightly loaded sectors. Accordingly, the users in the coordination region of a highly loaded sector can get more service from the relatively less loaded ones.

\section{B. Scenario 2 vs. scenario 3}

Figure 5 illustrated the performance in terms of flow throughput when four carriers are available in the system.

The results confirm our expectation in the sense that it is shown that the average throughput achieved according to scenario 2 (baseline MF+CA) is less important than that with the classical 4C-HSDPA corresponding to scenario 3.

Indeed, we see that the throughput of users in the coordination zone $\left(Z_{12}\right.$ for instance) decreases rapidly and reaches 0 at almost $4000 \mathrm{Kbits} / \mathrm{s}$ of traffic demand. From that point, users in the coordination zone will be accumulated, blocking by this way the set of resources $F_{c}$ while users in the non-coordination zone will be served with the remaining frequencies $F \backslash F_{c}$.

Accordingly, their corresponding throughput will decrease linearly as a classical 2C HSDPA system with a longer relative stability since only those users in the non-coordination zone 


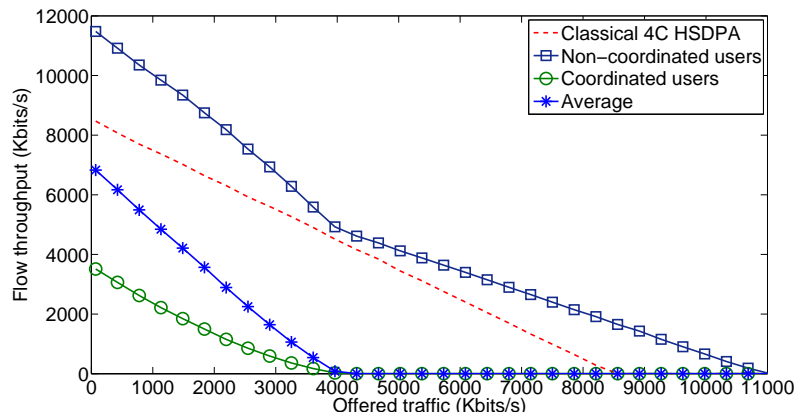

Fig. 5. Flow throughput (scenario 2 vs. scenario 3).

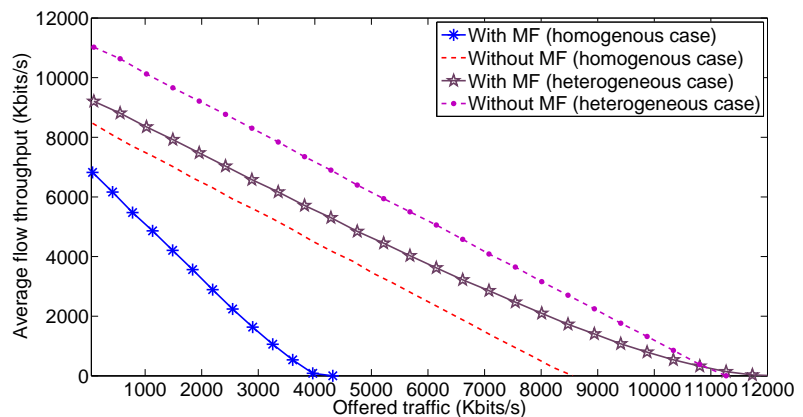

Fig. 6. Average throughput under different load conditions (scenario 2).

are eligible for scheduling. In summary, in this case, it is more efficient to deactivate the multi-flow operation and to deploy only carrier aggregation.

In order to investigate the multi-flow ability to balance load, we consider the case of heterogeneously loaded sectors. The average flow throughputs for different load conditions are given in figure 6. Although the system stability is improved when the neighboring sectors are less loaded, the performance, in average, remains much more important with a classical 4FHSDPA (scenario 3).

\section{Scenario 4 performance analysis}

In order to get full benefit from sector coordination in a network where several carriers can be aggregated, we should release the restriction on the number of frequencies that can be used to serve users in the coordination zone which is set to two carriers maximum while the system can support up to four carriers.

In figure 7, we plot the average throughput according to scenario 4 where multi-flow transmission is deployed on all the available carriers. We see that when sectors are equally loaded, coordination is beneficial only at low load. However, when neighboring sectors are lightly loaded, performing multiflow operation enables to offload the traffic across sectors; hence, the system stability is improved considerably.

\section{CONCLUSION}

We showed in this paper that multi-flow transmission performance depends on the network configurations and the load

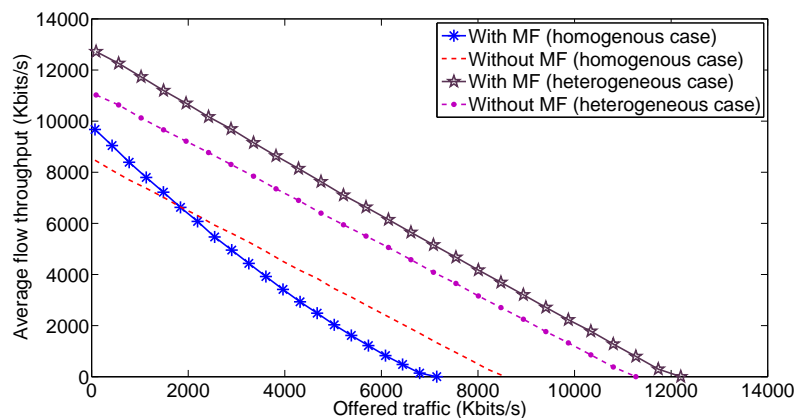

Fig. 7. Average throughput under different load conditions (scenario 4).

conditions.

Using Markovian analysis combined with network simulations, we showed that the multi-flow operation is highly recommended in single-carrier HSDPA system in order to boost throughput at cell edge and to balance load across nonuniformly loaded sectors.

However, when four carriers are aggregated, the multi-flow operation is not beneficial in average since it does not achieve any gain over the classical 4C-HSDPA system. This is due to the actual status of the standard that limits the number of carriers that can be used for the multi-flow transmission to two.

Releasing this restriction enables to get full benefit from multi-flow transmission and carriers aggregation in terms of throughput and load balancing.

\section{REFERENCES}

[1] K. Johansson, J. Bergman, D. Gerstenberger, M. Blomgren, and A. Walln, "Multi-carrier hspa evolution.," in VTC Spring, IEEE, 2009.

[2] "The evolution of HSPA: The 3GPP standards progress for fast mobile broadband using HSPA+," tech. rep., 4gamericas, October 2011.

[3] H. Holma and A. Toskala, WCDMA for UMTS: HSPA Evolution and LTE. New York, NY, USA: John Wiley \& Sons, Inc., 2007.

[4] T. Bonald, S.-E. Elayoubi, A. E. Falou, and J.-B. Landre, "Radio capacity improvement with hspa+ dual-cell.," in ICC, pp. 1-6, IEEE, 2011.

[5] A. A. Razzac, S.-E. Elayoubi, A. E. Falou, and B. E. Hassan, "Performance evaluation of dual carrier feature in the uplink of hspa+ systems.," in VTC Fall, pp. 1-5, IEEE, 2012.

[6] "3GPP TR 25.872: technical specification group radio access network; high speed packet access (HSDPA) multipoint transmission."

[7] "HSPA+ Advanced Smart Networks: Multipoint Transmission," tech. rep., Qualcomm Incorporated, February 2011.

[8] "R1-104913, multi-cell transmission techniques for HSDPA," tech. rep., NSN, Nokia, 3GPP 15 RAN1 62.

[9] D. Petrov, I. Repo, and M. Lampinen, "Overview of single frequency multipoint transmission concepts for HSDPA and performance evaluation of intra-site multiflow aggregation scheme," in VTC Spring, 2012, pp. $1-5$.

[10] A. Khlass, T. Bonald, and S. E. Elayoubi, "Flow-Level performance of intra-site coordination in cellular networks," in IEEE WiOpt, pp. 303310, May 2013.

[11] V. Hytonen, O. Puchko, T. Hohne, and T. Chapman, "Introduction of multiflow for HSDPA," in NTMS 2012, pp. 1-5.

[12] "Evolved universal terrestrial radio access (E-UTRA); further advancements for E-UTRA physical layer aspects (3GPP release9)."

[13] A. Saadani and J.-B. Landre, "Realistic performance of hsdpa evolution 64-qam in macro-cell environment.," in VTC Spring, IEEE, 2009.

[14] T. Bonald and M. Feuillet, Network Performance Analysis. ISTE/Wiley, July 2011. 